\title{
Teaching the millennial radiology resident: applying a five-step 'microskills' pedagogy
}

\author{
Colin Jingxian $\underline{\operatorname{Tan}}^{1}$, MBBS, FRCR, Chee Yeong $\underline{\operatorname{Lim}}^{1}$, MBBS, FRCR
}

\begin{abstract}
Radiology is a unique medical specialty that focuses on image interpretation and report generation with limited patient contact. Resident read-out sessions with teaching are a quintessential part of reporting workflow practices in teaching institutions. However, most radiologist-educators do not have formal training in teaching and learning experiences vary. The five-step 'microskills' model ('one-minute preceptor' technique) developed by Neher is an easily adopted teaching model that complements the workflow of the typical read-out session, and can be utilised by radiologists of varied teaching experience and seniority. The steps are: (a) get a commitment; (b) probe for supporting evidence; (c) teach general rules; (d) reinforce what was done right; and (e) correct mistakes. Feedback is important to the model and accounts for two out of five microskills. The teaching model emphasises knowledge application and establishing relevance, which is useful in engaging the millennial resident. It is easily assimilated and applied by radiologist-educators.
\end{abstract}

Keywords: education, microskills, radiology, residency

\section{INTRODUCTION}

The Singapore national medical specialty training programme has transitioned from an apprenticeship system into a highly structured model accredited by the American Council for Graduate Medical Education (International). ${ }^{(1)}$ This presents an opportunity for educational reform that equips the teaching faculty with effective pedagogical techniques for a new generation of trainees.

Most of our clinicians do not undergo formal training as educators and rely on personal experience in teaching. Furthermore, our public healthcare institution staff face a heavy clinical workload, limiting the time available for dedicated tutoring. As such, educational encounters with trainees are often less than ideal. The fast-paced mindset of the millennial resident poses a separate challenge for the faculty. As described by Slanetz et $\mathrm{al}_{,}{ }^{(2)}$ the stereotypical millennial grows up in the Internet era with information on demand and demonstrates a short attention span, preferring concise teaching sessions and collaborative learning. This contrasts with educators who are accustomed to traditional top-down, didactic approaches. Therefore, there is a need to develop efficacious, modern approaches to training in our hospitals.

\section{RADIOLOGY TRAINING CONCEPTS}

Diagnostic radiology (DR) is a unique specialty in which daily work centres on workstation image analysis and there is limited patient contact, precluding the application of patient-centred teaching pedagogies. Teaching occurs routinely during the daily read-out session: the resident reviews scan images side-by-side with a consultant, with an emphasis on abnormality detection and interpretation. The outcome is a draft radiological report, which is subsequently verified by the consultant. Selected educational techniques must thus be able to integrate with this workflow and mirror the structure of an ideal radiology report for efficiency and ease of adoption.

\section{FIVE-STEP 'MICROSKILLS' MODEL}

A widely accepted method that can be adapted for DR is the five-step 'microskills' model by Neher et al, ${ }^{(3)}$ also known as the 'one-minute preceptor' technique.(4) Originally conceptualised in 1980, ${ }^{(5)}$ it describes teaching behaviours linked to five dominant clinical teaching modes of conceptualisation, problemsolving, teacher-learner relations, feedback and role modelling scholarship. Neher et $\mathrm{al}^{(3)}$ adapted some of these teaching behaviours to design the following stepwise technique: (a) get a commitment; (b) probe for supporting evidence; (c) teach general rules; (d) reinforce what was done right; and (e) correct mistakes (Fig. 1).

The tasks can be considered as discrete components that may be reordered by the educator, hence allowing trainees to benefit from completed segments even if the teaching session is interrupted. This format also facilitates the preceptor's detection of knowledge gaps that can be addressed before further discussion. The model is intended to be highly efficient in a time-sensitive environment, with the original publication stating that the entire encounter can be completed within five minutes. The microskills also correspond well with the components of a radiology report and can be effortlessly incorporated into the read-out session.

In a typical reporting workflow, if the resident has already produced a draft report, it can be evaluated by the preceptor before the review encounter. Verbal prompting specific to each microskill is only required for a further question or to address a deficiency. Particularly relevant to Microskills 1 and 2, this can save valuable time for use in the subsequent discussions.

${ }_{1}^{1}$ Department of Diagnostic Radiology, Singapore General Hospital, Singapore

Correspondence: Dr Colin Jingxian Tan, Senior Resident, Department of Diagnostic Radiology, Singapore General Hospital, Outram Road, Singapore 169608. colin.tan.jingxian@gmail.com 


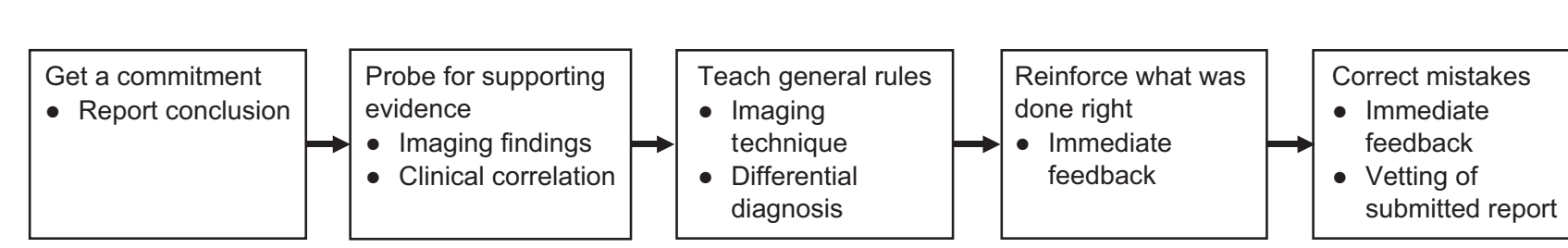

Fig. 1 Diagram shows the five-step 'microskills' method ${ }^{(5)}$ and how it corresponds to the radiological report and read-out discussion.

\section{Microskill 1: get a commitment}

After reading the images, the preceptor should encourage the trainee to propose a subjective conclusion for the study, beyond a mere description of abnormalities. This prompts the resident to analyse the imaging findings and make a tertiary level commitment, instilling the independent thinking required for first-line overnight on-call duties and eventual specialist practice. As shown in the COVER survey, ${ }^{(6)} 46.2 \%$ of clinicians "usually only read the conclusion of the radiology report" and thus, training on how to formulate a concise, clinically relevant conclusion is crucial. Novice residents may need to be prompted with questions such as 'What do you think are the major relevant imaging findings?' They may not be confident to venture a diagnosis, particularly if they are still developing core observational skills. Advanced learners, however, may initiate the discussion with a commitment to the diagnosis. In such instances, the preceptor should challenge the resident to make a higher-level interpretative commitment. For example, if the resident detects a hepatocellular carcinoma in the liver, the preceptor may follow up with a question on resectability.

\section{Microskill 2: probe for supporting evidence}

The next microskill is intuitive: the preceptor asks the trainee to substantiate his commitment with imaging evidence, corresponding to the descriptive findings section of the radiology report. This presents an opportunity for the educator to assess the knowledge and thought process of the trainee. New residents may again require direct prompting or redirection if their reasoning was incorrect. They can be called upon to explain specific features of the visualised pathology, such as the reasoning behind a 'silhouette' sign. An experienced trainee can be challenged in the realm of techniques and differential diagnoses, such as 'Which magnetic resonance $(\mathrm{MR})$ imaging sequences were key to establishing your diagnosis?' The discussion can also encompass relevant clinical information that contributes to the radiological diagnosis, such as serum alpha-fetoprotein levels in a suspicious hepatic lesion.

\section{Microskill 3: teach general rules}

By this stage, the clinical tutor should have identified the learner's depth of knowledge and maturity of analytical reasoning. While many preceptors may be tempted to launch into didactic teaching to impart theoretical knowledge, this may be ineffective in time-pressed workflows; furthermore, residents often find it challenging to assimilate more than a handful of key points per case. The tutor should instead aim to teach general rules or crucial learning points of the case instead. Broad principles applicable to other cases with similar imaging appearances are highlighted to the trainee as 'take home messages'. For example, using the case example of an MR imaging liver study using liver-specific contrast medium, the radiologist may teach the resident a general rule about lesion characteristics on the hepatobiliary phase. The approach should be concise, such as a focused discussion tabulating common hepatic lesions, hepatocyte content and their corresponding enhancement characteristics, to facilitate recall and future application.

This microskill is relevant to the findings section of the radiology report. In particular, the resident may have gleaned additional information that can be used to describe relevant negatives in the report.

\section{Microskills 4 and 5: reinforce what was done right, correct mistakes}

The two final microskills may be considered together as an exercise in giving feedback. The significance of feedback can be seen from the fact that it accounts for two out of five steps of this teaching method. In an in-depth article on the topic, $\operatorname{Archer}^{(7)}$ suggested some key guiding principles in the delivery of feedback: (a) feedback should be focused on the task rather than the individual; (b) it should be specific, even to the point of simple verification (correct vs. incorrect); (c) there should be a linkage to personal goals; and (d) feedback should be balanced and neither undermine self-esteem nor consist solely of praise. The third point requires elaboration: feedback recipients have intangible goals along the continuum of 'learning' and 'performance'. The former goal is associated with the desire to acquire new skills in order to improve intelligence. The latter performance-oriented group may believe that intelligence is innate and thus seeks to actively demonstrate competence to others, craving positive evaluation. Unlike the former group, they may shy away from challenging tasks and are more likely to give up. If a trainee is assessed to be overly performance-oriented during the interaction, the preceptor should aim to drive the trainee towards a learning orientation by breaking down complex tasks and subtly weighting positive feedback.

During the read-out session, the preceptor is next to the resident and has the opportunity to provide immediate verbal feedback. The educator must seize the chance to give feedback on the written report draft, with the discussion directed towards reporting structure and language. Assessment of the residents' understanding and assimilation of the discussion points would be based on the final draft report that the preceptor receives for verification. 
To reinforce what was done right means to present the learner with positive feedback for an appropriate action with the intention of establishing it as a competency. For example, the preceptor may tell the resident 'It is good that you delved into the clinical details of the patient. The fact that the patient has known chronic hepatitis $\mathrm{C}$ strengthens our provisional diagnosis of a hepatocellular carcinoma.' With this feedback, the preceptor hopes that the review of patients' clinical information becomes routine for every future scan that the learner encounters.

Correcting mistakes requires tact and a non-judgemental approach. The focus should not be on the error itself, but rather measures to avoid it in the future. Common mistakes in the radiology reporting setting include failure of detection or misinterpretation of imaging findings. The educator must advise how to improve on this, for example, by helping the resident define review areas for similar images in the future. Errors in report phrasing, structure or conclusion can be reworded by the educator on the spot for immediate feedback.

\section{DISCUSSION}

The five-step 'microskills' model of clinical teaching is well established as an effective teaching method for different levels of teaching experience and medical vocations. ${ }^{(8,9)}$ It can be a core component in a resident-teacher training curriculum, which some radiology residency programmes have started with favourable results. ${ }^{(10)}$ The key benefits of this method of teaching include the ease of assimilation, short time required per teaching encounter and seamless integration with the conventional radiology reporting workflow of side-by-side checking. The first three microskills also specifically correspond to relevant sections of the radiology report.

The role of the radiologist continues to evolve beyond the written report. ${ }^{(11)}$ Most radiologists are integral participants in multidisciplinary teams. The verbal sparring and on-the-fly analytical skills that are developed through the microskills method will serve the resident well when contributing to clinicoradiological rounds. These will also be useful for residents attempting oral or viva examinations.

\section{CONCLUSION}

To educate the millennial resident, the preceptor needs to transition from the traditional role as a subject matter expert to that of an educational facilitator who stimulates and guides discussion. The preceptor must teach the application of knowledge to live cases, and firmly establish the relevance and value of newly taught knowledge in day-to-day reporting. The five-step 'microskills' model encompasses these principles by design and is thus suitable for widespread adoption by radiologist-educators.

\section{REFERENCES}

1. Yang $\mathrm{H}, \mathrm{Tan} \mathrm{CJ}$, Lau DA, et al. Competency-based radiology residency: a survey of expectations from Singapore's perspective. Ann Acad Med Singapore 2015; 44:98-108

2. Slanetz PJ, Kung J, Eisenberg RL. Teaching radiology in the millennial era. Acad Radiol 2013; 20:387-9.

3. Neher JO, Gordon KC, Meyer B, Stevens N. A five-step "microskills" model of clinical teaching. J Am Board Fam Pract 1992; 5:419-24.

4. Neher JO, Stevens NG. The one-minute preceptor: shaping the teaching conversation. Fam Med 2003; 35:391-3.

5. Koen FM, Vivian AS. Learning the skills of clinical pharmacy teaching. Am J Pharm Educ 1980; 44:61-5

6. Bosmans JM, Weyler JJ, De Schepper AM, Parizel PM. The radiology report as seen by radiologists and referring clinicians: results of the COVER and ROVER surveys. Radiology 2011; 259:184-95.

7. Archer JC. State of the science in health professional education: effective feedback. Med Educ 2010; 44:101-8.

8. Bott G, Mohide EA, Lawlor Y. A clinical teaching technique for nurse preceptors: the five minute preceptor. J Prof Nurs 2011; 27:35-42.

9. Furney SL, Orsini AN, Orsetti KE, et al. Teaching the one-minute preceptor. A randomized controlled trial. J Gen Intern Med 2001; 16:620-4.

10. Donovan A. Radiology resident teaching skills improvement: impact of a resident teacher training program. Acad Radiol 2011; 18:518-24.

11. Knechtges PM, Carlos RC. The evolving role of the radiologist within the health care system. J Am Coll Radiol 2007; 4:626-35. 